
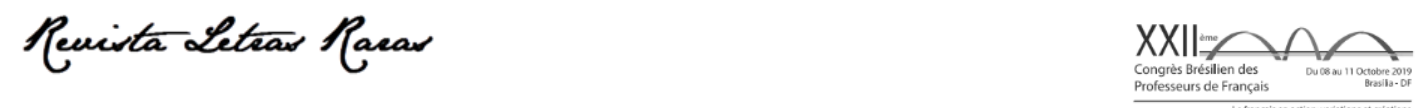

Actes du XXIlème Congrès brésilien des professeurs de français (dans Édition spéciale de la Revue Letras Raras 2020)

ISSN : 2317-2347 - v. 9, Dossier Spécial (2020)

Todo o conteúdo da RLR está licenciado sob Creative Commons Atribuição 4.0 Internacional

\title{
Les contradictions des politiques linguistiques brésiliennes et leurs enjeux pour une formation plurilingue et relativiste
}

\section{Andréia Matias Azevedo*}

Doctorat en Lettres dans le domaine de l'enseignement-apprentissage du FLE à l'Université Fédéral de Rio de Janeiro et travaille comme professeure de Français dans la Fundação de Apoio à Escola Técnica do Estado do Rio de Janeiro (FAETEC).

https://orcid.org/0000-0001-7854-1729

Reçu le 01 nov. 2020. Approuvé le 17 nov. 2020.

\section{Comment citer cet article :}

AZEVEDO, Andréia Matias. Les contradictions des politiques linguistiques brésiliennes et leurs enjeux pour une formation plurilingue et relativiste. In: CONGRES BRESILIEN DES PROFESSEURS DE FRANÇAIS, 22., 2019, Brasília. Actes du XXIIème Congrès Brésilien des Professeurs de Français. Édition spéciale de la Revue Letras Raras: Campina Grande. EDUFCG. nov. 2020, p. 440-453.

\section{RÉSUMÉ}

Cette étude vise à soulever une réflexion sur les politiques linguistiques brésiliennes concernant l'enseignement et l'apprentissage des langues étrangères au cours de l'histoire afin de démontrer que les actions du gouvernement brésilien vont à contre-courant des demandes du contexte politique, économique et social, favorisant ainsi la marche en arrière du pays. D'emblée, cette affirmation part de la nouvelle Base Nationale Commune Curriculaire Brésilienne (BNCC) dans laquelle on remarque que l'anglais est la seule langue étrangère enseignée. II ne reste aucun doute que l'anglais joue un rôle important dans l'actuel scénario ; pourtant, il serait judicieux également que le système éducatif offre d'autres langues de sorte que les apprenants puissent visionner le monde de différentes perspectives et, par conséquent, développent un sentiment d'altérité, et un regard critique sur l'Autre et Soi-même (LEVINAS, 2014 ; CARLO, 1998). Un autre point à remarquer touche le développement du pays dans la mesure où cette politique monolinguiste se montre nocive au projet d'internationalisation du système universitaire brésilien.

MOTS-CLÉS : Politiques linguistiques ; Monolinguisme ; Plurilinguisme ; Enjeux.

\section{Introduction}

De nombreuses études anthropologiques présentent le Brésil comme la nation de la multiculturalité et de la pluriculturalité. En effet, on constate dans ce pays un foisonnement culturel et ethnique, compte tenu du fait que cette nation s'est bâtie sur des rencontres des Indiens, des Portugais, des Africains, des Italiens, des Allemands, parmi d'autres. À l'instar de certains 

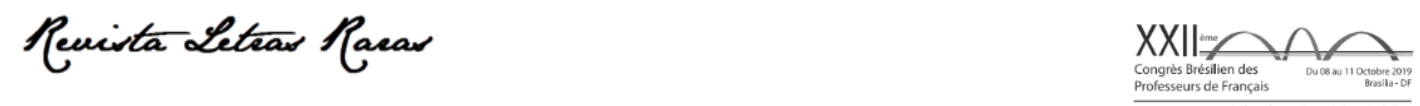

Actes du XXIlème Congrès brésilien des professeurs de français (dans Édition spéciale de la Revue Letras Raras 2020) ISSN : 2317-2347 - v. 9, Dossier Spécial (2020)

Todo o conteúdo da RLR está licenciado sob Creative Commons Atribuição 4.0 Internacional

spécialistes, Gilvan Müller de Oliveira (2009) souligne que l'on y parle plus de 215 langues ; pourtant, ces divers parlers ne s'intègrent pas à la société.

En parallèle, il vaut la peine de souligner qu'un nombre peu expressif de cette population maîtrise l'anglais, l'espagnol, le français et d'autres langues étrangères, vues comme fondamentales pour le marché du travail, l'internationalisation du pays et pour la formation du sentiment de l'altérité chez les individus.

Comme le remarque Aline Gohard-Radenkovic (1999), le monolinguisme et la monuculturalité nous remettent donc à repenser les principes d'universalisme culturel, la culture comme modèle de civilisation, et à l'Etat-Nation. À vouloir mieux comprendre les enjeux de ce monolinguisme brésilien, cet article portera sur l'enseignement-apprentissage des langues étrangères (LE), en particulier du français en cours de l'histoire.

Pour ce faire, nous nous appuierons sur des recherches dans les domaines pédagogique, sociolinguistique, historique et des études empiriques. Suite à cela, nous estimons pouvoir visionner des stratégies et des brèches pour revitaliser l'enseignement-apprentissage du Français Langue Etrangère (FLE) sachant que le public brésilien a encore un ou plusieurs intérêts à l'apprendre.

\subsection{Les enjeux des interventions monolinguistes et plurilinguistes}

Faut-il proposer une éducation centrée sur des principes monolinguistes ou plurilinguiste ? Bien que ce questionnement ne date pas de ce jour, il convient de dire qu'il génère encore des polémiques parmi de nombreux chercheurs et enseignants dans la mesure où ces orientations ont affaire à un certain positionnement politique. Dans ce cas, il s'avère judicieux que l'on analyse les enjeux sous-jacents à ces deux courants en tenant compte de la matérialité historique et social du scénario actuel.

Quant au monolinguisme proprement dit, il renvoie à réfléchir tout d'abord sur le mythe de la tour de Babel, dans lequel on prônait une langue unique en vue d'éviter des conflits entre les peuples. Par contre, le plurilinguisme remet aux divers parlers, à l'idéologie relativiste qui l'emporte sur celle d'ordre universaliste. Or, si l'on se penche sur ce sujet de façon moins manichéenne, il est possible de constater que les pays européens plurilingues vivent aussi des tensions entre langue(s) nationale(s) et celles édifiées en tant qu'officielle(s).

Comme le remarque Louis-Jean Calvet (2007), les hommes se sont toujours mis à intervenir sur les langues pour le compte de leur bon usage, de les normatiser et d'évincer leurs 

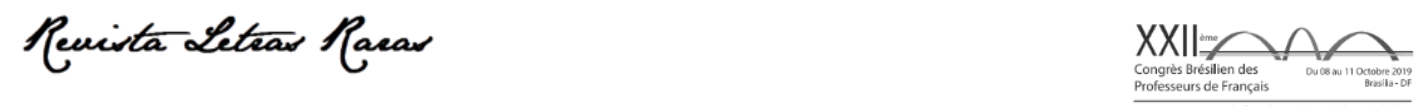

Actes du XXIlème Congrès brésilien des professeurs de français (dans Édition spéciale de la Revue Letras Raras 2020) ISSN : 2317-2347 - v. 9, Dossier Spécial (2020)

Todo o conteúdo da RLR está licenciado sob Creative Commons Atribuição 4.0 Internacional

imprécisions. Toutefois, il souligne que ces actions linguistiques obtiennent une autre dimension au fur et à mesure que l'Etat privilégie l'une langue au détriment de l'autre. Sur ce point, Calvet fait mention du concept de language planning de Einar Haugen, à travers lequel le chercheur démontre les interventions de l'État pour construire une identité nationale. Encore dans ce sillage, l'auteur ajoute que les études de Ferguson et de J. Fishman ont favorisé l'avènement de la sociolinguistique appliquée dans un contexte où divers pays étaient déjà libres du système colonial.

En vue d'attirer l'attention sur la tradition d'interventionnisme linguistique de la France, Marie-Josée de Saint Robert (2000) fait allusion à ces trois moments de l'histoire : en 1539, l'ordonnance de Villers-Cotterêts, un document dans lequel est autorisée l'emploi des langues maternelles pour les procédures législatives par conséquent, le latin perd de l'espace dans cet univers) ; en 1635, Richelieu fonde l'Académie française, une institution dont le rôle consistait notamment à normaliser et à perfectionner la langue française ; en 1635, apparaît la loi du 2 thermidor an II, un décret imposant le français comme l'unique langue administrative.

Par rapport au Brésil, Gilvan Müller de Oliveira (2009) affirme que les Etats portugais et brésilien ont adopté comme langue légitime le portugais. Selon l'auteur, ce pays a vécu une sorte de répression linguistique et de " glotocide ». Tout d'abord, il fait allusion à un document, intitulé Diretório dos Indios, dans lequel le marquis de Pombal établit des normes civilisatrices, parmi lesquelles figure la langue portugaise.

Ce faisant, le tupi, langue vernaculaire, subit une première attaque, malgré la résistance des Indiens. Encore sur les interventions linguistiques brésiliennes, Gilvan porte sur le régime dictatorial de Getúlio Vargas, pendant lequel des langues d'immigration telles que l'allemand et l'italien ont été interdites.

S'agissant des politiques linguistes adoptées au Brésil, le point suivant sera consacré à une analyse de nature historiographique de l'enseignement-apprentissage de langues étrangères (LE), en particulier, de la langue française. Pour cela, cet article se penchera notamment sur les directives et les orientations éducatives brésiliennes françaises à l'aide des études pionnières de Valnir Chagas (1979) et de Christian Puren (1988) ainsi que des documents tels que les PCNs, LDB et CECR.

\subsection{L'historiographie de l'enseignement-apprentissage des langues étrangères au} Brésil. 

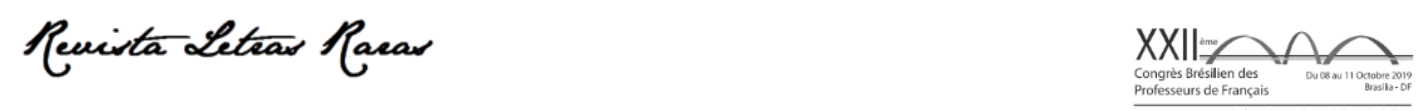

Actes du XXIlème Congrès brésilien des professeurs de français (dans Édition spéciale de la Revue Letras Raras 2020) ISSN : 2317-2347 - v. 9, Dossier Spécial (2020)

Todo o conteúdo da RLR está licenciado sob Creative Commons Atribuição 4.0 Internacional

Selon Valnir Chagas (1979), les premières actions qui se rapportent à l'enseignementapprentissage des LE au Brésil datent des années 1808, pendant le gouvernement de D. João VI. Quant à la langue moderne enseignée, à cette époque, on a choisi le français qui y jouissait d'un fort prestige social. Néanmoins, l'auteur remarque que son apprentissage ne devient obligatoire qu'en 1837, avec la fondation du Collège Pedro II.

À partir de 1855, le gouvernement propose la modernisation du curriculum afin de répondre aux besoins d'une société pré-républicaine. À ce propos, il convient de dire que l'éducation au Brésil, axée pendant longtemps sur les directives humanistes des jésuites, se base dès lors sur les principes de la philosophie positiviste. En ce qui concerne les LE, le français, l'anglais et l'allemand obtiennent le même statut de langues classiques.

Avec la création du ministère de l'éducation en 1930, le président Getúlio Vargas envisage, pour son programme de reconstruction nationale, la démocratisation de l'enseignement public brésilien. Dans ce scénario, le français et l'anglais comptent environ huit heures hebdomadaires dans le curriculum alors que le temps réservé au latin se restreint à six heures.

Mais l'enseignement des LES atteint son point culminant en 1942 pendant la période de la Reforme Capanema. Selon le ministre de l'Éducation et de la Santé, Gustavo Capanema, l'éducation devrait développer des habilités et des valeurs afin d'éveiller chez les sujets des sentiments nationaliste et humaniste. Ce faisant, l'apprentissage des langues étrangères ne visait pas l'ínternationalisation du pays et n'avait pas la prétention d'aller vers l'Autre, mais vers SoiMême. D'ailleurs, avec l'avènement du régime dictatorial de Getúlio Vargas, l'interdiction des langues italienne et allemande dans les systèmes éducatifs et dans les moyens communicatifs démontre effectivement que ses politiques linguistiques étaient ancrées sur des principes monolinguistes.

Avec la perte de prestige des langues d'immigration dans le territoire brésilien, il s'avère pertinent de mentionner le décret 389 de 1938, dans lequel on édicte que l'une des exigences pour accorder à quelqu'un la nationalité brésilienne est la connaissance de la langue portugaise. Dans cette conception, le gouvernement de Getúlio Vargas s'est engagé à mettre fin au travers de décrets les postes de professeurs de français, d'anglais et d'allemand du Collège Pedro II.

En 1950, les débats sur l'enseignement au Congrès National se centraient notamment sur la démocratisation de l'enseignement brésilien, compte tenu du fait que le pays avait besoin de gens plus scolarisés pour travailler dans les industries. Pour ce faire, beaucoup d'intellectuels, 

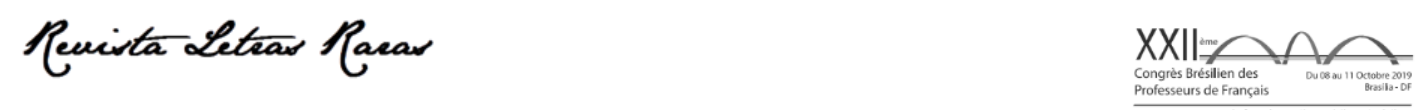

Actes du XXIlème Congrès brésilien des professeurs de français (dans Édition spéciale de la Revue Letras Raras 2020) ISSN : 2317-2347 - v. 9, Dossier Spécial (2020)

Todo o conteúdo da RLR está licenciado sob Creative Commons Atribuição 4.0 Internacional

d'éducateurs et d'hommes politiques revendiquaient la création de la Loi des Directives et Bases pour l'Education (LDB), dont l'intention principale était de proposer un système éducatif plus unifié.

Toutefois, cette loi ne s'est concrétisée qu'en 1961 et s'est bâtie plutôt sur une philosophie libéraliste, garantissant une forte présence du secteur privé. Vilson Leffa (1999) remarque que ces nouvelles directives établissent une réduction de l'enseignement des LE de près de $70 \%$ en comparaison à la période du gouvernement Capanema.

Pour rappel, après la Seconde Guerre Mondiale, la relation entre les Etats-Unis et le Brésil se renforce dans le domaine économique et culturel. Dans les institutions scolaires, le prestige de la langue anglaise s'impose sur celui de la langue française. En accord avec la LDB de 1961, les états ont eu la liberté de choisir les LEs qui seraient dispensées au collège et au lycée. Notons que certains établissements scolaires ont décidé de les enlever de leur curriculum.

Toutefois, pour faire face à l'expansion de la culture et de la langue anglaise dans le scénario international, Daniel Coste (1976) affirme que le gouvernement français s'est mis à investir de manière intensive dans les programmes culturels et éducatifs dans l'extérieur.

Pour revenir aux politiques brésiliennes, pendant le régime militaire, l'éducation technocratique l'emporte sur celle d'ordre humaniste. Au lieu de cours de langues et de philosophie, on confère aux élèves des cours professionnels et on leur enseigne des disciplines, telles que "Éducation Morale et Civique", visant à leur imposer des valeurs morales.

Dans ce contexte, on peut pointer également que l'enseignement privé se répand encore plus dans la mesure où l'investissement dans l'enseignement public se restreint à moins de $3 \%$ du PIB. À partir de la LDB de 1996 (loi 9.394), l'enseignement des LE redevient obligatoire dans le curriculum. Or, le nombre important d'étudiants en classe ne contemplant que deux séances hebdomadaires ne permet pas aux enseignants de travailler les quatre activités langagières (parler, écrire, lire et écouter) et de les sensibiliser aux aspects culturels. Par rapport aux LE optionnelles, ce défi augmente de manière significative pour l'enseignant car il est censé dispenser en cinquante minutes un cours dans une situation similaire et avoir, par exemple, deux fois plus de journaux de classes et/ou d'évaluations à corriger.

Actuellement, la Base Nacional Curricular (BNCC) ne propose que l'enseignement de l'anglais, comme nous l'avons déjà souligné. Maints éducateurs et enseignants se montrent contre cette mesure car elle va à l'encontre du plurilinguisme et du pluriculturalisme. Au niveau des directives éducatives brésiliennes, on ne peut se passer de citer aussi les Parâmetros Curriculares 

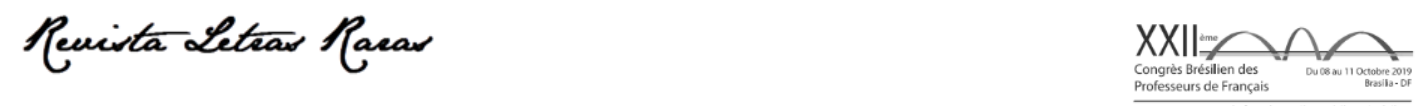

Actes du XXIlème Congrès brésilien des professeurs de français (dans Édition spéciale de la Revue Letras Raras 2020) ISSN : 2317-2347 - v. 9, Dossier Spécial (2020)

Todo o conteúdo da RLR está licenciado sob Creative Commons Atribuição 4.0 Internacional

Nacionais (PCNs, 2002), un document dans lequel on trouve des orientations afin d'aider les enseignants, les éducateurs et tous ceux qui travaillent dans ce domaine.

À l'heure qu'il est, ils considèrent donc judicieux que les apprenants sachent se servir, dans leur vie personnelle et professionnelle, des nouvelles ressources technologiques et scientifiques. Dans le milieu des LEs, on constate dans les PCNs toute une préoccupation de proposer une formation axée sur des principes pluriculturels et plurilingues en vue de répondre aux besoins d'un monde mondialisé. Dans ce sillage, l'enseignement-apprentissage des LE centré pendant longtemps sur des aspects linguistiques envisage en ce moment une formation plus complexe.

Avec l'intention de mieux visionner l'espace des LEs, en particulier du français, dans le système éducatif brésilien en ce moment, il semble judicieux de définir également les rôles de la municipalité, de l'État et du Gouvernement Fédéral dans les prochaines lignes.

Face à cela, nous porterons sur les politiques linguistes dans ces trois sphères sur la base d'articles, de documents et d'études de cas. Au terme de la présentation de ces données, nous viserons à les analyser et à soulever de nouvelles réflexions sur les politiques linguistiques brésiliennes auprès des enseignants de français, des institutions et de tous ceux qui s'intéressent à l'apprentissage et conçoivent les LEs comme fondamentales à la formation des sujets au niveau ontologique, mais aussi, à leur "devenir".

\section{Les politiques des langues étrangères dans l'État de Rio}

Si l'on se penche proprement dit sur l'enseignement de la langue française, on identifie que le nombre d'étudiants de français dans les écoles publiques de l'État et de la municipalité de Rio se réduisent fortement. Cela est dû au manque de concours adressés aux enseignants de français, dès qu'ils obtiennent leur retraite, on met à la place du français l'anglais. Bien que ce type de conception et de pratiques d'ordre monolinguiste et monoculturiste l'emporte sur celles axées sur des principes plurilinguistes et pluriculturels, on visionne également l'avènement de quelques écoles bilingues dans la municipalité de Rio.

En effet, les évènements internationaux dans l'État, comme la Journée de la Jeunesse en 2013, la Coupe du Monde en 2014 et les Jeux Olympiques en 2016 ont favorisé l'intérêt du maire Eduardo Paes pour mettre en oeuvre le Programme Rio, Enfant Global (le décret 31187 de 

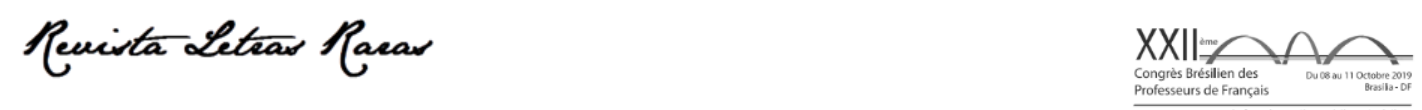

Actes du XXIlème Congrès brésilien des professeurs de français (dans Édition spéciale de la Revue Letras Raras 2020) ISSN : 2317-2347 - v. 9, Dossier Spécial (2020)

Todo o conteúdo da RLR está licenciado sob Creative Commons Atribuição 4.0 Internacional

06/10/2009). À Rio, en 2015, la municipalité de Niterói a inséré le français dans cinq écoles du réseau grâce au partenariat entre l'Université Fédéral Fluminense, l'Alliance Française de Niterói et l'Association des Professeurs de Français de l'Etat de Rio (APFERJ) et le gouvernement local.

En 2013, le gouvernement de l'État a créé le programme Enseignement Secondaire Interculturel visant à offrir des formations en mandarin, français, espagnol, anglais et en turc dans cinq écoles de ce réseau. L'intention principale de ce projet consiste à conférer aux apprenants une formation en LE qui les rendent capables de s'exprimer et de faire la connaissance d'autres cultures de manière plus significative.

Pour rappel, l'enseignement-apprentissage des LE se centre en général, dans les écoles brésiliennes, sur la compréhension écrite. Dans le cas spécifique du français, la formation bilingue portugais/français des apprenants du CIEP 449 (Centros Integrados de Educação Pública) à Niterói vient d'un partenariat entre l'Etat de Rio et l'Académie de Créteil, comptant sur le soutien du Consulat Général de France à Rio. Outre ces deux instances, l'Alliance Française de Niterói participe par la prise en charge de la formation des enseignants de cette école.

Dans la sphère de l'État, on ne peut se passer de mentionner la Fundação de Apoio à Escola Técnica (Faetec). D'emblée, il s'avère essentiel de souligner que cette institution éducative appartient au Secrétariat des Science et Technologie de Rio et est chargé de l'éducation professionnelle depuis 1997. Ses cours et ses formations sont gratuits et couvrent tous les niveaux : l'enseignement fondamental, secondaire technique et supérieur.

Au regard de l'apprentissage des LE, Faetec offre également des cours d'idiomes adressés à la communauté et aux apprenants de l'institution, contemplant trois langues étrangères : anglais, espagnol et français.

Étant professeur de français de cette institution dans le quartier d'Ipanema, il faut dire que le public de français est très diversifié. D'un côté, nous avons des étudiants avec des objectifs spécifiques ayant besoin d'apprendre le français en fonction de leur travail et de leurs études, d'autres veulent parler une autre langue et connaître d'autres cultures.

Bien que l'équipe de Français ait adopté une méthode avec des objectifs plus larges, rien n'empêche que l'on se serve de temps en temps de méthodes du Français de Spécialité (FS) conçues pour des groupes composés de professionnels du même domaine. Par rapport au Centro de Educação Tecnológico Profissionalizante (CETEP) à Ipanema, il faut dire que maintes personnes travaillent dans le secteur de l'hôtellerie-restauration. 

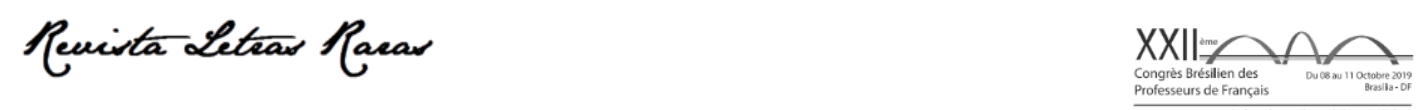

Actes du XXIlème Congrès brésilien des professeurs de français (dans Édition spéciale de la Revue Letras Raras 2020) ISSN : 2317-2347 - v. 9, Dossier Spécial (2020)

Todo o conteúdo da RLR está licenciado sob Creative Commons Atribuição 4.0 Internacional

Face à ce panorama, il est possible de constater que Faetec a une philosophie de formation qui répond hautement aux besoins de la Base Nacional Curricular (BNCC), dans la mesure où elle propose des formations techniques, professionnelles et académiques à plein temps. En ce qui concerne les classes de langues, comme l'on avait explicité, les apprenants du secondaire ont la possibilité d'étudier trois LEs: le français, l'anglais et l'espagnol. Notons que ces directives sur la manière de traiter les LEs, favorisant le contact des apprenants avec diverses langues et cultures, s'alignent sur les principes des PCNs, du CECR, des études récentes et du contexte politico-économique-social dans lequel nous vivons.

En revanche, comme l'Université de l'Etat de Rio (UERJ), Faetec a fait face à un moment assez difficile. Avec la crise de Rio, les centres de l'institution n'avaient pas capacité d'assurer les services fondamentaux, comme les services de nettoyage et d'entretien.

En analysant le nombre de candidats inscrits aux cours de langues d'Ipanema, on constate qu'il y a encore un fort intérêt pour l'apprentissage du français. Selon les informations de l'institution, 152 personnes se sont inscrites en espagnol ; 310, en anglais et 217, en français.

Selon les informations des coordinateurs et des directeurs, entre autres, l'institution ne proposera plus de concours aux enseignants de français. D'ailleurs, beaucoup de centres de langues n'ont plus de cours de français.

À partir de cette historiographie de l'enseignement des LE, en particulier du français, nous pouvons conclure que le gouvernement fédéral ne visionne pas de propositions, de projets concernant la formation plurilingue et pluriculturelle aux niveaux de l'enseignement fondamental et du secondaire.

Au contraire, la nouvelle BNCC, selon les études présentées, ne reconnaît que l'anglais comme LE. Un tel choix se justifie principalement par l'importance de cette langue sur le marché mondial. Par ailleurs, en filigrane à cela, il est encore commun que les économies plus développées imposent également leurs cultures sur celles qui se présentent plus fragiles.

Sur cette ligne d'analyse, il convient d'ajouter que l'investissement de la Banque mondial (BIRD) dans l'éducation brésilienne a été accordée sous certaines conditions. À savoir, il faut que le gouvernement propose des formations aux professeurs, offrent des écoles à plein temps et des formations techniques et professionnelles. Au demeurant, il importe de préciser que cet organisme conçoit l'éducation au travers d'une optique néolibéraliste (AKKARI; SANTIAGO, 2012)

\subsection{Le recul des politiques éducatives et linguistiques}



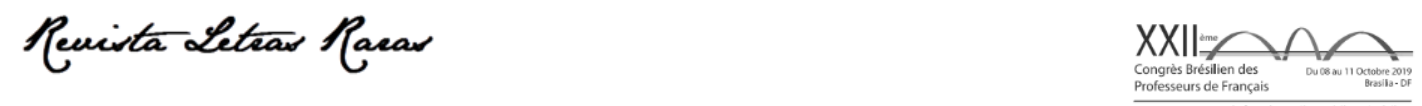

Actes du XXIlème Congrès brésilien des professeurs de français (dans Édition spéciale de la Revue Letras Raras 2020) ISSN : 2317-2347 - v. 9, Dossier Spécial (2020)

Todo o conteúdo da RLR está licenciado sob Creative Commons Atribuição 4.0 Internacional

En analysant les projets de l'éducation brésilienne au cours de l'histoire, on remarque qu'elle se centre notamment sur une politique néolibérale. Cela signifie que l'on vise notamment à former des sujets pour le marché du travail, capables de produire et de consommer. Quant au rôle de l'État brésilien, il convient de rappeler qu'il ne se limite qu'à maintenir le bon fonctionnement de ce système. Dans le cas du Brésil, l'action néolibérale devient encore plus nocive lorque que l'Etat essaie d'échapper à sa responsabilité sociale en conférant au secteur privée des services tels que l'éducation et la santé.

Notons que les gouvernements des présidents Luís Inácio Lula da Silva et Dilma Rousself se sont aussi basés sur une politique néolibéraliste ; toutefois, l'État s'est mis à s'occuper de l'éducation et à mettre en place politiques sociales. À savoir, de nouvelles universités publiques ont été créées et du programme PROUNI, concédant des bourses aux étudiants dans l'enseignement supérieur privé.

Pour rappel, avec le programme Science sans Frontières en 2011 (CAPES ; CNPQ, 2011, p. 1), il y a eu un fort sentiment de revitalisation de la langue française. Par exemple, à l'Alliance Française de Rio, de nombreux étudiants universitaires se sont inscrits aux cours, car ils avaient envie notamment de partir en France et au Canada.

Quant aux universités publiques, le programme Science sans Frontières a mené à l'avènement de nouvelles recherches afférentes à l'enseignement du Français sur Objectif Universitaire (FOU).

En outre, le programme Science Sans Frontières a également contribué à la création du programme Idiomes Sans Fronteira (IsF). En effet, le gouvernement s'est rendu compte que le système éducatif brésilien avait négligé au cours de l'histoire de l'enseignement de LE. Ainsi, pour améliorer les compétences des étudiants dans ce domaine, le gouvernement s'est engagé à proposer des cours de langues à distance.

Or, avec la fin du gouvernement Dilma Rousself, le programme Science Sans Frontières semble aussi avoir pris fin. Pour cela, le Ministère de l'Éducation soutient que la raison est que cette initiative n'a pas apporté les résultats attendus. Comme l'un des motifs, le ministre de l'Education Mendonça Filho fait mention principalement du bas niveau d'anglais des étudiants

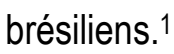

\footnotetext{
${ }^{1}$ https://oglobo.globo.com/sociedade/educacao/especialistas-concordam-com-fim-do-programa-ciencia-semfronteiras-21149172, le 18 avril 2018.
} 

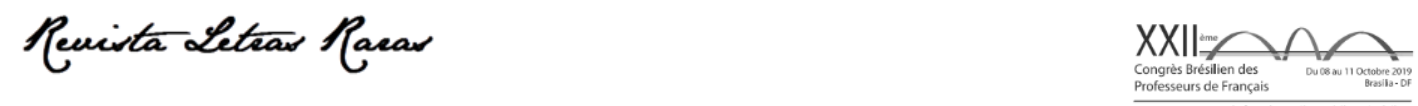

Actes du XXIlème Congrès brésilien des professeurs de français (dans Édition spéciale de la Revue Letras Raras 2020)

ISSN : 2317-2347 - v. 9, Dossier Spécial (2020)

Todo o conteúdo da RLR está licenciado sob Creative Commons Atribuição 4.0 Internacional

Outre l'achèvement de ce programme, le gouvernement Fédéral génère en ce moment une forte réduction budgétaire dans le secteur social. Avec l'instauration d'un amendement constitutionnel, intitulé PEC 241, l'actuelle équipe politique établit une limite pour les dépenses publiques. Cela représente la fin des investissements publics dans l'éducation de ces vingt dernières années.

\section{CONSIDÉRATION FINALE}

Pour contrecarrer les attaques du gouvernement envers le plurilinguisme et la langue française dans les écoles publiques, il semble que la meilleure stratégie consiste à promouvoir des partenariats avec des universités étrangères et des institutions qui s'intéressent à la langue française et souhaitent former des sujets francophones, capables d'agir comme médiateurs de la culture française, canadienne et sénégalaise entre autres. Pour rappel, il est important de citer les partenariats établis entre l'État de Rio, l'École de Créteil, l'Alliance Française de Rio et le Consulat Français.

On reconnaît que le principal vecteur de motivation du gouvernement brésilien en ce moment est d'ordre économique. Autrement dit, l'objectif de ce type de partenariat se centre surtout sur la possibilité de trouver des moyens pour réduire les dépenses et former des sujets au marché du travail. Toutefois, nul ne questionne le fait que cela favorisera les sujets à avoir une formation de nature plus humaniste et transcendant ainsi les intérêts du système capitaliste.

Un autre point fondamental consiste à promouvoir des politiques valorisant les enseignants de langue étrangère. Tout d'abord, il est judicieux que l'on voie ce professionnel comme un intellectuel qui est capable de pousser les sujets à la réflexion critique. On ne peut négliger le fait que l'enseignement des LEs est censé avoir des connaissances communicatives et noncommunicatives au minimum provenant de deux univers. 

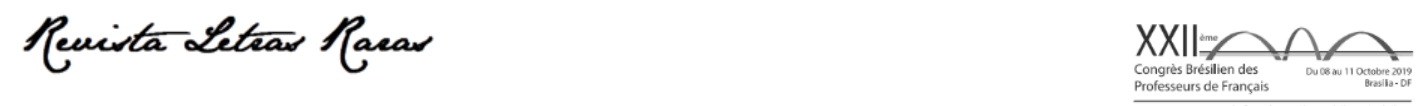

Actes du XXIlème Congrès brésilien des professeurs de français (dans Édition spéciale de la Revue Letras Raras 2020) ISSN : 2317-2347 - v. 9, Dossier Spécial (2020)

Todo o conteúdo da RLR está licenciado sob Creative Commons Atribuição 4.0 Internacional

\section{Références}

ALBURQUEQUE-COSTA, H. "Formation aux savoir-faire académiques dans une université brésilienne", dans PARPETTE, C. et MANGIANTE, J.-M. (dir), Faire des études supérieures en langue française, Le Français dans le monde. Recherches, 2016.

AKKARI, A. ; SANTIAGO, M. « L'impact des politiques néolibérales sur l'éducation : tensions entre prise en compte des diversités et standardisation », Carrefours de l'éducation 2012/2 ( $n^{\circ} 34$ ), p. $77-94$.

AKKARI, A. Inégalités éducatives structurelles au Brésil : Entre État, privatisation et décentralisation. Carrefours de l'éducation, no 8, 1999, p. 140-162.

ALVES, J. A terceirização do ensino de línguas estrangeiras em escolas de ensino formal. São Paulo: USP, 2010.

BNCC. Disponible :

http://portal.mec.gov.br/index.php?option=com_docman\&view=download\&alias=79601-anexotexto-bncc-reexportado-pdf-2\&category_slug=dezembro-2017-pdf\&ltemid=30192. Accès le 04 mai 2018.

BRASIL. Parâmetros Curriculares Nacionais: terceiro e quarto ciclos do ensino fundamental Língua Estrangeira. Secretaria de Educação Fundamental. Brasília: MEC/SEF, 1998.

BRASIL. Secretaria de Educação Média e Tecnologia. Parâmetros Curriculares Nacionais: ensino médio, Ministério da Educação, Secretaria de Educação Média e Tecnológica. Brasília: MEC/Semtec, 2002.

BRASIL. Senado Federal. (1996). Lei de Diretrizes e Bases da Educação Nacional: nº 9394/96. Brasília.

CALVET, L.-J. La sociolinguistique. Paris, P.U.F, 1993.

CALVET, L.-J. As políticas lingüísticas. Tradução Jonas Tenfen e Marcos Bagno. São Paulo: Parábola, 2007.

CAMPOS, C. M. A política da língua na era Vargas: proibição do falar alemão e resistências no sul do Brasil. Disponíble dans le site http://www.bibliotecadigital.unicamp.br/document/?down=000135324. Accès le 15 jan. 2018.

Campus France. Disponible dans le site : <www.brasil.campusfrance.org.> Accès le 04 mai. 2018.

CHAGAS, Valnir. (1979) Didática especial de línguas modernas. 3. ed. São Paulo: Companhia Editora Nacional.

Ciência Sem Fronteira. Disponible dans le site : https://oglobo.globo.com/sociedade/educacao/especialistas-concordam-com-fim-do-programaciencia-sem-fronteiras-21149172. Accès le 31 avril 2018.

CONSEIL DE L'EUROPE. Un Cadre européen Commun de Référence pour les langues : apprendre, enseigner, évaluer. Paris : Éditions Didier, 2000.

Consulado-geral da França no Rio de Janeiro. Escola pública bilíngue é inaugurada em Niterói. Disponíble dans le site : http://riodejaneiro.ambafrancebr.org/ESCOLAPUBLICA-BILINGUE-E accès le 5 mail. 2018. 

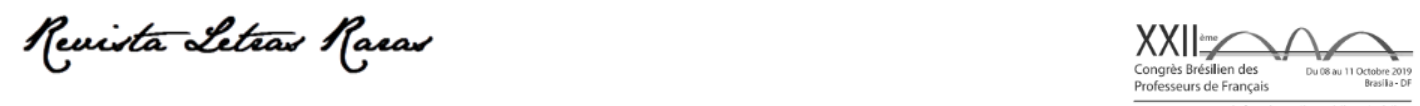

Actes du XXIlème Congrès brésilien des professeurs de français (dans Édition spéciale de la Revue Letras Raras 2020) ISSN : 2317-2347 - v. 9, Dossier Spécial (2020)

Todo o conteúdo da RLR está licenciado sob Creative Commons Atribuição 4.0 Internacional

COOPÉRATION CULTURELLE - Comité de l'Éducation. Un Cadre Européen Commun de Référence pour les Langues : apprendre, enseigner, évaluer. Strasbourg : Didier, 2005.

COSTE, D. Un niveau seuil. Le Français dans le Monde, 1976.

Council of Europe. Plurilingual Education in Europe: 50 years of international cooperation. Council of Europe, Language Policy Division. Strasbourg, 2006. Disponible sur le site : http:www.coe.int/t/dg4/linguistic/Source/PlurilingualEducation_EN.pdf . Accès le 7 out. 2017.

Educação \& Realidade, Porto Alegre, v. 39, n. 4, p. 1137-1152, out./dez, 2014. Disponible dans le sitel : http://www.scielo.br/pdf/edreal/v39n4/10.pdf

GARCIA, N. " Monolinguisme politique dans une société plurilingue ? Le cas du Luxembourg », Revue internationale de politique comparée, vol. 21, p. 17-36, 2014. DOI 10.3917/ripc.214.0017

GOULART, N. Escolas internacionais: por que Fuvest e Enem não são alvos? Disponível em: http://veja.abril.com.br/educacao/escolas-internacionais-porque- fuvest-e-enem-nao-sao-osalvos/ . Accès le 15 out. 2017

GOHARD-RADENKOVIC, A. Communiquer en langue étrangère : de compétences culturelles vers des compétences linguistiques. Bern, Berlin, Bruxelles, Frankfurt/M., New York, Wien : Peter Lang, 1999.

IBGE. Censo 2000. Disponible dans le site : http://portalgeo.rio.rj.gov.br/estudoscariocas/download/2394_\%C3\%8Dndice\%20de\%20Desenvol vimento\%20Social_IDS.pdf. Accès le 12 avril 2018.

LEVINAS, E. Totalidade e infinito. Tradução de José Pinto Ribeiro. 3. ed. Lisboa : Edições 70, 2014.

MANGIANTE, J.-M. ; PARPETTE, C. Le Français sur Objectif Universitaire. Grenoble : PUG, 2011. MEC. (2013). Ministerio da Educação. Consulté le 04 mai 2018, dans le site http://portal.mec.gov.br/default.htm

MEC. (2013) . Ministério da Educação. Consulté le 02 mai 2018, dans le site http://portal.mec.gov.br/component/tags/tag/34913

MÉSZÁROS, I. A educação para além do capital. Tradução de Isa Tavares. 2. ed. São Paulo: Boitempo, 2008

MORIN, E. Os sete saberes necessários à educação do futuro. São Paulo: Cortez, 2000

MOURA, Selma. (2009). A. Educação bilíngue no Brasil: possibilidades e desafios rumo a uma sociedade linguística e culturalmente plural. Dissertação (Mestrado Disponible dans le site: http://www3.fe.usp.br/secoes/semana08/completos/103.swf . Accès le : 14 jan. 2017.

MOURA, Sema. (2010). O que é uma escola internacional? Disponible dans le site:https://educacaobilingue.com/2016/10/11/o-que-e-uma-escola internacional/. Accès le 3 mars 2018.

OCDE. Quelle part de leur richesse nationale les pays consacrent-ils à l'éducation ? Paris : Éditions OCDE, $2010 \mathrm{~b}$.

OCDE. Perspectives de l'emploide l'OCDE.Paris : Éditions OCDE, 2011. 

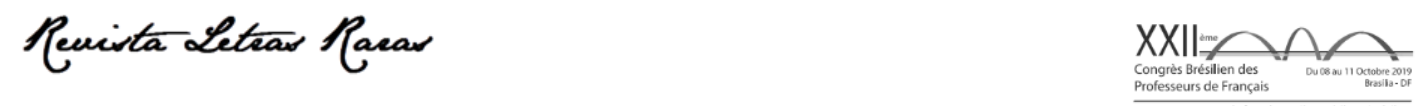

Actes du XXIlème Congrès brésilien des professeurs de français (dans Édition spéciale de la Revue Letras Raras 2020) ISSN : 2317-2347 - v. 9, Dossier Spécial (2020)

Todo o conteúdo da RLR está licenciado sob Creative Commons Atribuição 4.0 Internacional

OCDE. Toujours plus d'inégalité : pourquoiles écarts de revenus se creusent. Paris : Éditions OCDE, 2012.

OLIVEIRA, G. M. O lugar das línguas: a América do Sul e os mercados linguísticos na nova economia. In : STREHLER, R. ; GOROWITZ, S. (Org.). Politiques publiques et changements en éducation : pour un enseignement réciproque du portugais et du français. Synergies Brésil, São Paulo, número especial, 2010.

PARÂMETROS CURRICULARES NACIONAIS. In

http://portal.mec.gov.br/seb/arquivos/pdf/pcn_estrangeira.pdf. Accès le 21 avril 2013.

Plurilinguismo no Brasil: repressão e resistência. In: DAHLET, V. B. (Org.). Le Brésil et ses langues : perspectives en français. Synergies Brésil, São Paulo, número 7, 2009.

PEREIRA, Telma C. (2006). O ensino de línguas estrangeiras como um fator de inclusão social : o desafio da francofonia no Rio de Janeiro. Tese (Doutorado) - Pontifícia Universidade Católica do Rio de Janeiro, Rio de Janeiro.

PIETRARÓIA, Christina. (2006). "Le français instrumental à l'aube du XXI e siècle" in XV e Congrès des professeurs de français. Plurilinguisme et identité culturelle. Belo Horizonte - 9/13 octobre 2005.

PNE - Plano Nacional de Educação. Disponible dans le site : $<$ http://portal.mec.gov.br/index.php?option=com_content\&view=article\&id=16478\&ltemid=1107>. Accès en : out. 2017.

PUREN, C. Continuités, ruptures et circularités dans l'évolution de la didactique des langues étrangères en France. Études de Linguistique Appliquée, Paris: Didier Érudition, n. 78, p. 65-74, abr.jjun., 1990.

PUREN, C. Histoires des méthodologies de l'enseignement des langues. Paris: Nathan/CLE International, 1998.

PUREN, C. La didactique des langues-cultures entre la centration sur l'apprenant et l'éducation transculturelle. Paris: APLV, jun. 2008. Disponível em: <www.aplv-languesmodernes.org>. Acèss le 10 juil. 2008.

Prefeitura de Niterói. Disponible dans le site http://www.niteroi.rj.gov.br/index.php?option=com_content\&view=article\&id=1867:niteroi-tera-aprimeira-escola-publica-bilingue-do-brasil Accès le 5 nov. 2017.

Quartiero E.-M., Bianchetti, L. (Orgs.). (2005). Educação corporativa. Mundo do trabalho e do conhecimento: Aproximações. São Paulo: Cortez; Santa Cruz do Sul : Editora da UNISC. Santos M. Por uma outra globalização: do pensamento único à consciência universal. Rio de Janeiro: Record, $3^{\mathrm{a}}$ ed., 2000.

RELATÓRIO para a Unesco da Comissão Internacional sobre Educação para o Século XXI. Educação: um tesouro a descobrir. 4. ed. São Paulo: Cortez; Brasília: MEC/Unesco, 2000.

ROCHA, Luana. (2016) 0 ensino de língua estrangeira em Niterói: um olhar político linguístico. Niterói: UFF.

ROY, J.-L. Qual o futuro da língua francesa? Francofonia e concorrência cultural no século XXI? Porto Alegre : Ed. Sulinas, 2010. 

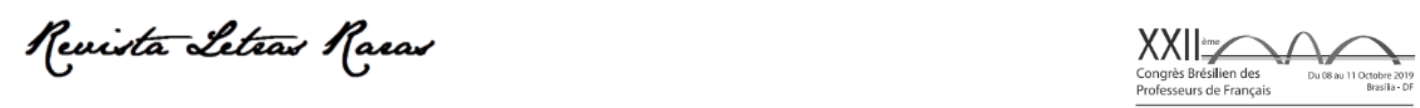

Actes du XXIlème Congrès brésilien des professeurs de français (dans Édition spéciale de la Revue Letras Raras 2020)

ISSN : 2317-2347 - v. 9, Dossier Spécial (2020)

Todo o conteúdo da RLR está licenciado sob Creative Commons Atribuição 4.0 Internacional

Saint-Robert, Marie-Josée de. Que sais-je ? La Politique de la langue française : Presses Universitaires de France: Paris, 2000.

Seeduc. Disponible dans le site : http://www.ri.gov.br/web/seeduc/exibeconteudo?articleid=2509691. Accès le 30 avril 2018.

VALLE, I. R. ; BIANCHETTI, L. «Éducation et recherche au Brésil : du projet nationaliste à la globalisation », Carrefours de l'éducation 2012/2 (n³4), p. 61-76.DOI 10.3917/cdle.034.0061

Vasconcellos, Maria Drosila. (2001). Les politiques éducatives au Brésil : des pionniers à nos jours. Revue Tiers Monde, 42(167), pp. 657---672.

WCEFA - CONFERÊNCIA MUNDIAL DE EDUCAÇÃO PARA TODOS. (1990). Declaração mundial sobre educação para todos e Plano de ação para satisfazer as necessidades básicas de aprendizagem. Jomtien, Tailândia, mar. 